\title{
Homogeneous Riemannian manifolds with generic Ricci tensor
}

\author{
by WŁodZimierz JeloneK (Kraków)
}

\begin{abstract}
We describe homogeneous manifolds with generic Ricci tensor. We also prove that if $\mathfrak{g}$ is a 4 -dimensional unimodular Lie algebra such that $\operatorname{dim}[\mathfrak{g}, \mathfrak{g}] \leq 2$ then every left-invariant metric on the Lie group $G$ with Lie algebra $\mathfrak{g}$ admits two mutually opposite compatible left-invariant almost Kähler structures.
\end{abstract}

0. Introduction. In this paper, we study Riemannian homogeneous manifolds $(M, g)$ which admit certain totally geodesic invariant foliations with leaves locally isometric to a Lie group with a left-invariant metric. In particular, we study Riemannian homogeneous manifolds whose Ricci tensor is generic, i.e. whose Ricci tensor has $n=\operatorname{dim} M$ different eigenvalues. We also give some criteria for a homogeneous manifold to be locally isometric to a Lie group $(G, g)$ with a left-invariant metric $g$. In [B-V] very interesting examples are presented of 4 -dimensional homogeneous $\mathcal{A}$-manifolds with generic Ricci tensor. These examples are unimodular Lie groups. In the present paper we prove that every simply connected homogeneous $\mathcal{A}$ manifold with generic Ricci tensor has to be a unimodular Lie group with a left-invariant metric, and that such manifolds cannot be geodesic orbit (g.o.) spaces. In particular, there are no naturally reductive homogeneous spaces with generic Ricci tensor. We also prove that if the Ricci tensor of a homogeneous g.o. space is negative definite, then all its eigenvalues have multiplicity grater than or equal to 2. Furthermore, we show that homogeneous spaces with generic Ricci tensor can admit left-invariant almost Kähler structures.

W. Thurston's $[\mathrm{T}]$ famous example (first given by Kodaira [Kod]) of a symplectic manifold which does not admit any Kähler structure, admits a structure of almost Kähler manifold which is a compact quotient of a leftinvariant homogeneous almost Kähler structure on a 4-dimensional nilpo-

2000 Mathematics Subject Classification: 53C30, 53C15, 53D05.

Key words and phrases: generic Ricci tensor, Kähler structure, almost Kähler structure, 4-dimensional Lie group, geodesic orbit space. 
tent Lie group with a left-invariant metric. We generalize these examples and prove that if $G$ is a 4-dimensional Lie group with unimodular Lie algebra $\mathfrak{g}$ such that $\operatorname{dim}[\mathfrak{g}, \mathfrak{g}] \leq 2$, then every left-invariant metric on $G$ admits a compatible almost Kähler structure. We also give simple examples of locally homogeneous Riemannian metrics with generic Ricci tensor on the torus $T^{4}$ which admit two mutually opposite invariant almost Kähler structures. Such examples are interesting in connection with the Goldberg conjecture (see [Se-1], [Se-2], [O-S], [C-F-L], [F-G]). Still unresolved, the Goldberg conjecture says that every compact Einstein almost Kähler manifold must be Kähler. Note that the Goldberg conjecture comes from Riemannian geometry rather than from symplectic geometry, i.e. one can imagine that there exists a compact Kähler manifold $(M, \omega)$ with Kähler form $\omega$ such that $(M, \omega)$ has an associated strictly almost Kähler structure $(M, g, J)$ and $(M, g)$ is Einstein.

At present there are many examples of strictly almost Kähler structures on compact 4-dimensional manifolds (see e.g. [O-T]); recently Drăghici has given a large class of symplectic 4-manifolds $(M, \omega)$ such that $\omega$ is not a Kähler form of any Kähler structure $(M, g, J)$ on $M$ (see [D]). However they are given in a non-explicit form and it is difficult to check whether $(M, \omega)$ admits, for example, an associated almost Kähler structure $(M, g, J)$ such that $(M, g)$ has constant scalar curvature. Our examples show that the flat Einstein-Kähler metric $\left(T^{4}, g, J\right)$ on $T^{4}$ is not an isolated point in the space of Riemannian locally homogeneous almost Kähler metrics on $T^{4}$, i.e. there exists a smooth family $\left(T^{4}, g_{b}, J_{b}\right), b>0$, of different strictly almost Kähler locally homogeneous structures on $T^{4}$ (whose Kähler form comes from a flat Kähler metric on $T^{4}$ ), convergent as $b \rightarrow 1$ to the flat Einstein-Kähler metric $\left(T^{4}, g, J\right)$.

1. Preliminaries. Let $(M, g)$ be a smooth connected Riemannian space. We denote by $\operatorname{Iso}(M)$ the Lie group of all isometries of $(M, g)$. The group $\operatorname{Iso}(M)$ acts smoothly from the left on $(M, g)$. A group $G \subset \operatorname{Iso}(M)$ is called transitive if for all $x, y \in M$ there exists an isometry $\phi \in G$ such that $\phi(x)=y$. For a right-invariant vector field $X \in \mathfrak{g}$ we denote by $X^{*}$ the fundamental vector field of the action of $G$ corresponding to $X$, i.e. $X_{x}^{*}=\frac{d}{d t}\left\{t \mapsto a_{t} \cdot x\right\}$ where $a_{t}$ is the one-parameter subgroup of $X$.

Let $G, H$ be Lie groups with Lie algebras $\mathfrak{g}, \mathfrak{h}$ and $H \subset G$. The coset manifold $M=G / H$ is reductive if there is an $\operatorname{Ad}(H)$-invariant subspace $\mathfrak{m}$ of $\mathfrak{g}$ that is complementary to $\mathfrak{h}$ in $\mathfrak{g}$. A naturally reductive homogeneous space is a reductive coset manifold $M=G / H$ furnished with a $G$-invariant metric such that, for the corresponding scalar product $h$ on the space $\mathfrak{m}$,

$$
h\left([X, Y]_{\mathfrak{m}}, Z\right)=h\left(X,[Y, Z]_{\mathfrak{m}}\right)
$$


where $[X, Y]_{\mathfrak{m}}$ is the $\mathfrak{m}$-component of $[X, Y]$ with respect to the decomposition $\mathfrak{g}=\mathfrak{h} \oplus \mathfrak{m}$.

A Riemannian homogeneous space is called a g.o. (geodesic orbit) space (see $[\mathrm{K}-\mathrm{V}]$ ) if every geodesic $\gamma$ in $M$ is an orbit of a one-parameter subgroup of isometries of $M$, i.e. $\gamma(t)=\phi_{t}\left(x_{0}\right)$ where $\left\{\phi_{t}: t \in \mathbb{R}\right\} \subset \operatorname{Iso}(M)$ is a one-parameter subgroup of Iso $(M)$. Every naturally reductive homogeneous space is a g.o. space, but there are also many homogeneous g.o. spaces $(M, g)$ which are not naturally reductive (see $[\mathrm{Ka}])$. Let $\nabla$ be the Levi-Civita connection of $(M, g)$. The curvature tensor $R$ of $(M, g)$ is defined by

$$
R(X, Y) Z=\nabla_{X} \nabla_{Y} Z-\nabla_{Y} \nabla_{X} Z-\nabla_{[X, Y]} Z
$$

and the Ricci tensor $\varrho$ of $(M, g)$ is

$$
\varrho(X, Y)=\operatorname{tr}\{Z \mapsto R(Z, X) Y\} .
$$

By an $\mathcal{A}$-manifold we mean (after A. Gray) a $\operatorname{Riemannian~space~}(M, g)$ whose Ricci tensor $\varrho$ satisfies the condition $\nabla_{X} \varrho(X, X)=0$ for every $X \in$ $T M$. We have the well known

\section{Proposition 1. Every g.o. space is an $\mathcal{A}$-manifold.}

Proof. Let $\gamma$ be a geodesic on $M$ such that $\gamma(0)=x_{0}, \dot{\gamma}(0)=X$. Then $\nabla_{X} \varrho(X, X)=\left.\frac{d}{d t} \varrho(\dot{\gamma}(t), \dot{\gamma}(t))\right|_{t=0}$. Let $Y \in \mathfrak{i s o}(M)$ be a Killing vector field such that $Y \circ \gamma(t)=\dot{\gamma}(t)$. Then $\left.\frac{d}{d t} \varrho(\dot{\gamma}(t), \dot{\gamma}(t))\right|_{t=0}=Y \varrho(Y, Y)_{x_{0}}=0$.

Let $S$ be the Ricci endomorphism of $(M, g)$, i.e. $\varrho(X, Y)=g(S X, Y)$. Note that $S$ is self-adjoint with respect to $g$. It follows that $S$ is diagonalizable and has only real eigenvalues. The symmetric matrices with all eigenvalues distinct form a generic subset in the set of all symmetric matrices. We call a Riemannian homogeneous space $(M, g)$ a space with generic Ricci tensor if its Ricci tensor has $\operatorname{dim} M$ different eigenvalues. If $S$ is a Ricci tensor of a Riemannian homogeneous space then we denote by $\left\{\lambda_{1}, \ldots, \lambda_{k}\right\}$ $(k \leq n)$ the distinct eigenvalues of $S$ and by $D_{\lambda_{i}}=\operatorname{ker}\left(S-\lambda_{i} \operatorname{Id}\right\}$ the eigensubbundle corresponding to $\lambda_{i}$. Note that each $\lambda_{i}$ is constant, and $D_{\lambda_{i}}$ is a distribution of constant dimension. If $\mathfrak{g}$ is a Lie algebra then $\mathfrak{z}(\mathfrak{g})$ denotes the center of $\mathfrak{g}$, i.e. $\mathfrak{z}(\mathfrak{g})=\{X \in \mathfrak{g}:[X, Y]=0$ for all $Y \in \mathfrak{g}\}$.

We denote by $\mathfrak{X}(M)$ the Lie algebra of smooth vector fields on $M$. If $(M, g)$ is a simply connected, complete Riemannian manifold and $\mathfrak{g} \subset$ $\mathfrak{X}(M)$ is a $\operatorname{dim} M$-dimensional Lie algebra spanned by an orthonormal frame $\left\{E_{1}, \ldots, E_{n}\right\}, E_{i} \in \mathfrak{X}(M)$ (i.e. $E_{i} \in \mathfrak{g}$ and $\left[E_{i}, E_{j}\right]=\sum_{k=1}^{n} c_{i j}^{k} E_{k}$ where $c_{i j}^{k} \in \mathbb{R}$ ) then there exists a structure of a Lie group $G$ on $M$ such that $E_{1}, \ldots, E_{n}$ are left-invariant vector fields on $G$, and $g$ coincides with the left-invariant metric on $G$ given by the frame $\left\{E_{1}, \ldots, E_{n}\right\}$ (see $[\mathrm{T}-\mathrm{V}]$ ).

Assume that $X$ is a Killing vector field $(X \in \mathfrak{i s o}(M))$. This means that the endomorphism $\nabla X \in \Gamma\left(T M \otimes T M^{*}\right)$ defined by the formula $\nabla X(v)=$ 
$\nabla_{v} X$ is skew-symmetric. The following well known formula holds (see $[\mathrm{K}]$, $[\mathrm{K}-\mathrm{N}])$ :

$$
\frac{1}{2} \Delta\|X\|^{2}=\|\nabla X\|^{2}-\varrho(X, X),
$$

where $\Delta$ is the Laplace operator of $(M, g),\|X\|$ is the norm of $X$ and $\|\nabla X\|$ is the norm of the endomorphism $\nabla X: T M \ni v \mapsto \nabla_{v} X \in T M$.

Let $(M, g, J)$ be a 4-dimensional almost Hermitian manifold, i.e. $(M, g)$ is a Riemannian manifold, $J: T M \rightarrow T M$ is an almost complex structure $\left(J^{2}=-\mathrm{id}_{T M}\right)$ and $g(J X, J Y)=g(X, Y)$ for all $X, Y \in T M$. The Kähler form $\Omega \in \Gamma\left(\bigwedge^{2} M\right)$ of $(M, g, J)$ is defined by $\Omega(X, Y)=g(J X, Y)$. The Hodge star operator (see [De]) $*$ (which depends on the orientation of $M$ ) defines an endomorphism $*: \bigwedge^{2} M \rightarrow \bigwedge^{2} M$ with $*^{2}=$ id and we denote by $\wedge^{+}, \Lambda^{-}$its eigensubbundles corresponding to $1,-1$ respectively. Such manifolds are always oriented and we choose an orientation in such a way that $\Omega$ is a self-dual form (i.e. $\Omega \in \wedge^{+} M$ ).

An almost Hermitian manifold is called almost Kähler if $d \Omega=0$; the corresponding almost Hermitian structure is then called an almost Kähler structure. The Lee form $\theta \in \Gamma\left(T M^{*}\right)$ of an almost Hermitian 4-manifold $(M, g, J)$ is defined by the equality $d \Omega=\theta \wedge \Omega$. We have $\theta=-\delta \Omega \circ J$ (see [C-S-V]) where $\delta \Omega(X)=-\operatorname{tr}_{g} \nabla \cdot \Omega(\cdot, X)$. Recall here that for a tensor $\Phi \in \Gamma\left(\otimes^{m} T M^{*}\right)$ of type $(0, m), \Phi: T M \times \ldots \times T M \rightarrow \mathbb{R}$, we define

$$
\operatorname{tr}_{g \mid i, j} \Phi=\sum_{k=1}^{n} \Phi\left(X_{1}, \ldots, X_{i-1}, E_{k}, X_{i+1}, \ldots, X_{j-1}, E_{k}, X_{j+1}, \ldots, X_{m}\right)
$$

where $\left\{E_{1}, \ldots, E_{n}\right\}$ is any orthonormal frame on $M$ and $n=\operatorname{dim} M$. It follows that $(M, g, J)$ is an almost Kähler manifold if and only if $\delta \Omega=0$, which is equivalent to

$$
\delta J=0,
$$

where $\delta J \in \Gamma(T M)$ is defined by the formula $\delta J=-\operatorname{tr}_{g} \nabla J(\cdot, \cdot)$. In fact $\nabla_{X} \Omega(Y, Z)=g\left(\nabla_{X} J Y, Z\right)$, thus $\delta \Omega(X)=g(\delta J, X)$.

A Hermitian 4-manifold $(M, g, J)$ is said to have an opposite almost Kähler structure if it admits an almost Kähler structure $\bar{J}$ with anti-self-dual Kähler form $\bar{\Omega}$. Note that if $\Omega, \bar{\Omega}$ are two mutually opposite almost Hermitian structures on a 4-manifold $M$, then in the neighborhood of every point $x \in M$ there exists a local orthonormal frame $\left\{E_{1}, E_{2}, E_{3}, E_{4}\right\}$ such that $\Omega=E_{1} \wedge E_{2}+E_{3} \wedge E_{4}$ and $\bar{\Omega}=E_{1} \wedge E_{2}-E_{3} \wedge E_{4}$. Indeed, since $\Omega^{2}=2 v_{g}, \bar{\Omega}^{2}=-2 v_{g}$, where $v_{g}$ is the volume form of $(M, g)$, we have $\omega_{1}^{2}=0=\omega_{2}^{2}$, where $\omega_{1}=\frac{1}{2}(\Omega+\bar{\Omega})$ and $\omega_{2}=\frac{1}{2}(\Omega-\bar{\Omega})$. Since $* \omega_{1}=\omega_{2}$ and $g\left(\omega_{1}, \omega_{1}\right)=1=g\left(\omega_{2}, \omega_{2}\right)$, there exists a local orthonormal frame such that $\omega_{1}=E_{1} \wedge E_{2}$ and $\omega_{2}=E_{3} \wedge E_{4}$ (we identify $T M$ and $T M^{*}$ by means 
of $g$ ). This means that $J E_{1}=E_{2}, J E_{3}=E_{4}, \bar{J} E_{1}=E_{2}, \bar{J} E_{3}=-E_{4}$. If $M=G$ is a Lie group with a left-invariant metric and $J, \bar{J}$ are left-invariant, then clearly we can take a global left-invariant orthonormal frame with the above property.

2. Ricci tensors with unit Killing eigenfields. Let $(M, g)$ be a Riemannian homogeneous space and let $G \subset \operatorname{Iso}(M)$ be a transitive group of isometries of $(M, g)$. Define

$$
l(G)=\left\{X \in \mathfrak{X}(M):\left[X, Y^{*}\right]=0 \text { for all } Y^{*} \in \mathfrak{g}^{*} \subset \mathfrak{i s o}(M)\right\}
$$

where $Y^{*}$ is the fundamental vector field corresponding to $Y, \mathfrak{g}$ is the Lie algebra of the group $G$, and set

$$
l_{i}(G)=\left\{X \in l(G): S X=\lambda_{i} X\right\} .
$$

Theorem 1. Let $(M, g)$ be a Riemannian homogeneous space and let $S$ be its Ricci endomorphism. Let $G$ be a transitive group of isometries of $(M, g)$. Then:

(a) Every vector field $X \in l(G)$ is complete.

(b) $l(G)$ is a finite-dimensional Lie subalgebra of $\mathfrak{X}(M), \operatorname{dim} l(G) \leq n$, and $\mathfrak{z}(\mathfrak{g}) \subset l(G), \mathfrak{z}(\mathfrak{i s o}(M)) \subset l(G)$.

(c) For every $X, Y \in l(G)$ the function $g(X, Y)$ is constant on $M$; in particular, if $X \in l(G)$ and $X_{x_{0}}=0$ for some $x_{0} \in M$ then $X=0$ on $M$.

(d) The vector space $l(G)$ is the direct sum $l(G)=l_{1}(G) \oplus \ldots \oplus l_{k}(G)$ of the vector spaces $l_{i}(G)$.

(e) The distribution spanned by $l(G)$ is a totally geodesic foliation, i.e. if $X, Y \in l(G)$ then $[X, Y] \in l(G)$ and $\nabla_{X} Y \in l(G)$. Every leaf of the foliation $l(G)$ is locally isometric to a Lie group with a left-invariant metric.

(f) If additionally $(M, g)$ is a g.o. space and $G=\operatorname{Iso}(M)$ then $l(G) \subset$ $\mathfrak{i s o}(M), l(G)$ is the center of $\mathfrak{i s o}(M)$ and $\nabla_{X} Y=0$ for any $X, Y \in l(\operatorname{Iso}(M))$ $=\mathfrak{z}(\mathfrak{i s o}(M))$. If $l_{i}(G) \neq\{0\}$ then $\lambda_{i} \geq 0$.

Proof. (a) To prove (a) we can assume that $G$ is a connected group, otherwise we consider the identity component of $G$. Let $x_{0} \in M$ and let $\left\{\phi_{t}\right.$ : $|t|<\varepsilon\}$, where $\varepsilon>0$, be a local one-parameter group of transformations corresponding to $X$, defined in a neighborhood $V$ of $x_{0}$. Since $\left[X, Y^{*}\right]=0$ for every $Y \in \mathfrak{g}$, it follows that if $x \in V$, then $\phi_{t}\left(g_{s} x\right)=g_{s} \phi_{t}(x)$, where $\left\{g_{s}\right.$ : $s \in \mathbb{R}\} \subset G$ is a one-parameter subgroup corresponding to $Y$ considered as a right-invariant vector field on $G$. Since exp $: \mathfrak{g} \rightarrow G$ is a local diffeomorphism of a neighborhood of 0 onto a neighborhood $U$ of the identity $e$, it follows that $\phi_{t}(g x)=g \phi_{t}(x)$ for every $x \in V$ and $g \in U$. Hence the group $\left\{\phi_{t}:|t|<\varepsilon\right\}$ is defined on $U V$ and hence on $\bigcup_{n \in \mathbb{N}} U^{n} V$. Since $G$ is connected and acts transitively on $M$, we get $G=\bigcup_{n \in \mathbb{N}} U^{n}$ and consequently $\phi_{t}(g x)=g \phi_{t}(x)$ for every $g \in U$ and $x \in M$ if $|t|<\varepsilon$. Thus the one-parameter group 
$\left\{\phi_{t}:|t|<\varepsilon\right\}$ is defined on the whole of $M$. Now if $t \in \mathbb{R}$ take $n \in \mathbb{N}$ such that $|t / n|<\varepsilon$ and put $\phi_{t}=\phi_{t / n} \circ \phi_{t / n} \circ \ldots \circ \phi_{t / n}$. Then $\phi_{t}$ is a global one-parameter group corresponding to the field $X$.

(b)\&(c) If $X, Y \in l(G)$ then for any $Z \in \mathfrak{g}$ we have $[X, Z]=[Y, Z]=0$. Consequently, $[[X, Y], Z]=-[[Z, X], Y]-[[Y, Z], X]=0$, hence $[l(G), l(G)]$ $\subset l(G)$. Thus $l(G)$ is a Lie subalgebra of $\mathfrak{X}(M)$. Let $X, Y \in l(G)$ and $v \in T M$. Then there exists a fundamental vector field $Z \in \mathfrak{g}(M)$ such that $Z_{x_{0}}=v$. Consequently, $v g(X, Y)=L_{Z}(g(X, Y))_{x_{0}}=g([Z, X], Y)+$ $g(Y,[Z, Y])=0$. It follows that $g(X, Y)$ is constant for any $X, Y \in l(G)$. In particular any $X \in l(G)$ has a constant length $\|X\|=\sqrt{g(X, X)}$. Let $x_{0} \in M$ be an arbitrary point and define a linear mapping $F: l(G) \rightarrow T_{x_{0}} M$ by $F(X)=X_{x_{0}}$. Then $F$ is injective since $F(X)=0$ implies $\|X\|=$ $\|X\|_{x_{0}}=0$, thus $X=0$. It follows that $l(G)$ is finite-dimensional and $\operatorname{dim} l(G) \leq n=\operatorname{dim} M$.

(d) Note that if $X \in l(G)$, then $S X \in l(G)$, since for any $Z \in \mathfrak{g}$ we have $L_{Z}(S X)=\left(L_{Z} S\right) X+S\left(L_{Z} X\right)=0$. Consequently, $S: l(G) \rightarrow l(G)$ is a well defined endomorphism of $l(G)$ with eigenvalues belonging to the set $\left\{\lambda_{1}, \ldots, \lambda_{k}\right\}$. Thus $l(G)=l_{1}(G) \oplus \ldots \oplus l_{k}(G)$.

(e) Since for any infinitesimal isometry $X \in \mathfrak{i s o}(M)$ we have $L_{Z} \nabla=0$, it is clear that $L_{Z}\left(\nabla_{X} Y\right)=0$ for any $X, Y \in l(G)$. Consequently, $\nabla_{X} Y \in l(G)$ if $X, Y \in l(G)$.

Now, it is easy to show that every simply connected open subset $U \subset$ $L$ of any leaf $L$ of the foliation spanned by $l(G)$ is isometric to an open neighborhood of the identity element $e \in H$ of some simply connected Lie group $H$ with a left-invariant metric $m$.

(f) To prove (f), assume that $(M, g)$ is a homogeneous g.o. space and that $G=\operatorname{Iso}(M)$. Let $X \in l(G)$ and let $v \in T_{x_{0}} M$ be arbitrary. Let $\gamma$ be a geodesic such that $\gamma(0)=x_{0}$ and $\dot{\gamma}(0)=v$. Since $(M, g)$ is a g.o. space there exists a Killing vector field $Y \in \mathfrak{i s o}(M)$ such that $\gamma(t)=\phi_{t}\left(x_{0}\right)$ where $\phi_{t}$ is a one-parameter subgroup corresponding to the field $Y$. Note that $[X, Y]=0$ and consequently $\nabla_{X} Y=\nabla_{Y} X$. Thus we have

$$
g\left(\nabla_{v} X, v\right)=g\left(\nabla_{Y} X, Y\right)_{x_{0}}=g\left(\nabla_{X} Y, Y\right)_{x_{0}}=-g\left(\nabla_{Y} Y, X\right)_{x_{0}}=0
$$

since $\nabla_{Y} Y=0$ along $\gamma$. It follows that the operator $\nabla X: v \mapsto \nabla_{v} X$ is skew-symmetric, which implies that $X \in \mathfrak{i s o}(M)$. Now it is clear that $X \in \mathfrak{z}(\mathfrak{i s o}(M))$ and $l(G)=\mathfrak{z}(\mathfrak{i s o}(M))$. Any Killing vector field $X$ of constant length satisfies $\nabla_{X} X=0$. Thus for any $X, Y \in l(\operatorname{Iso}(M))=\mathfrak{z}(\mathfrak{i s o}(M))$ we have $\nabla_{X} Y+\nabla_{Y} X=0$ and consequently, since $\nabla_{X} Y-\nabla_{Y} X=[X, Y]=0$, we obtain $\nabla_{X} Y=0$.

Now assume that $l_{i}(\mathfrak{i s o}(M)) \neq\{0\}$. Then there exists a unit Killing vector field $X$ such that $S X=\lambda_{i} X$. From (1.1) it follows that $\varrho(X, X)=$ $\|\nabla X\|^{2}$. Consequently, $\lambda_{i}=\|\nabla X\|^{2} \geq 0$. 
Corollary 1. Assume that $(M, g)$ is a simply connected homogeneous g.o. space such that $\operatorname{dim} l(\operatorname{Iso}(M))=n=\operatorname{dim} M$. Then $(M, g)$ is isometric to the group $\left(\mathbb{R}^{n}\right.$, can) with its flat standard bi-invariant metric.

Proof. This is an immediate consequence of Theorem 1(f).

Let $(M, g)$ be a homogeneous space and $G$ be a transitive group of isometries of $(M, g)$. Denote by $l_{G}$ the dimension of $l(G)$.

Proposition 2. Let $(M, g)$ be a simply connected homogeneous space. Then $(M, g)$ is isometric to a Lie group $(G, m)$ with a left-invariant metric $m$ if and only if there exists a transitive group $H$ of isometries of $(M, g)$ with $l_{H}=n=\operatorname{dim} M$.

Proof. If $(M, g)$ is a Lie group with a left-invariant metric and $H$ is the group of all left translations of $M$ then it is clear that $l(H)$ is the Lie algebra of all left-invariant vector fields, hence the "if" implication follows.

Now assume that $H$ is a transitive group of isometries of $(M, g)$ such that $l_{H}=n$. Let $G$ be the simply connected Lie group corresponding to the Lie algebra $l(H)$. Since $M$ is simply connected and complete (as it is homogeneous), there is an isometry of $(G, m)$ onto $(M, g)$.

Now we give a characterization of homogeneous spaces whose Ricci tensor is generic, i.e has all eigenvalues distinct.

Proposition 3. Assume that a simply connected homogeneous space $(M, g)$ has a generic Ricci tensor. Then $l_{\operatorname{Iso}(M)}=n=\operatorname{dim} M$ and $(M, g)$ is isometric to a Lie group $(G, m)$ with a left-invariant metric. The space $(M, g)$ is not a g.o. space.

Proof. Since the Ricci tensor $S$ is generic, it has $n$ different eigenvalues $\left\{\lambda_{1}, \ldots, \lambda_{n}\right\}$ and the eigendistributions $D_{\lambda_{i}}$ are one-dimensional. Since $M$ is simply connected it is clear that each $D_{\lambda_{i}}$ is oriented. Let $\left\{E_{1}, \ldots, E_{n}\right\}$ be a global orthonormal frame on $M$ such that $S E_{i}=\lambda_{i} E_{i}$. We show that $l(\operatorname{Iso}(M))=\operatorname{span}\left\{E_{1}, \ldots, E_{n}\right\}$, i.e. $l_{i}(\operatorname{Iso}(M))=\mathbb{R}\left\{E_{i}\right\}$. Let $X \in \mathfrak{i s o}(M)$. Then $L_{X} S=0$ and consequently $S\left[X, E_{i}\right]=\lambda_{i}\left[X, E_{i}\right]$. On the other hand since $g\left(E_{i}, E_{i}\right)=1$ we have $g\left(\left[X, E_{i}\right], E_{i}\right)=0$. Thus $\left[X, E_{i}\right]=0$ for any $X \in \mathfrak{i s o}(M)$. It follows that $l_{i}(\operatorname{Iso}(M))=\mathbb{R}\left\{E_{i}\right\}$.

If $(M, g)$ were a g.o. space then from Corollary 1 it would have to be a flat space with $S=0$ (a contradiction).

REMARK. In $[\mathrm{B}-\mathrm{V}]$ it is proved in a slightly different way that a simply connected homogeneous space with generic Ricci tensor has to be a Lie group with a left-invariant metric.

Corollary 2. Assume that $(M, g)$ is a homogeneous space. Then on $(M, g)$ there exists an at least $\operatorname{dim} \mathfrak{z}(\mathfrak{i s o}(M))$-dimensional space $V \subset \mathfrak{X}(M)$ of Killing eigenfields of the Ricci tensor. If $M$ is a simply connected g.o. space 
and $\mathfrak{z}(\mathfrak{i s o}(M))=0$ then all the eigenvalues of $\varrho$ have multiplicity greater than or equal to 2. If $M$ is compact and $\mathfrak{z}(\mathfrak{i s o}(M)) \neq\{0\}$ then $\chi(M)=0$.

Proof. The first part is obvious. To prove the second part, assume that $(M, g)$ is a simply connected homogeneous g.o. space. Note that if $X \in$ $\mathfrak{z}(\mathfrak{i s o}(M)$ then $\|X\|$ is constant on $M$. Thus if $\mathfrak{z}(\mathfrak{i s o}(M)) \neq\{0\}$ and $M$ is compact then $\chi(M)=0$. If $\varrho$ admits an eigenvalue $\lambda$ of multiplicity one then there exists a unit eigenfield $X$ corresponding to $\lambda$. Clearly $X \in l_{\lambda}(\operatorname{Iso}(M))$ and consequently $X \in \mathfrak{z}(\mathfrak{i s o}(M))$. Thus if $\mathfrak{z}(\mathfrak{i s o}(M))=0$ then all eigenvalues have multiplicity greater than or equal to 2 .

Corollary 3. Assume that $(M, g)$ is a homogeneous g.o. space whose Ricci tensor is negative definite. Then $\mathfrak{z}(\mathfrak{i s o}(M))=0$ and every eigenvalue of $S$ has multiplicity greater than or equal to 2.

Proof. Note that if $\varrho$ had an eigenvalue $\lambda_{i}$ of multiplicity 1 , then $(M, g)$ (up to a two-fold covering) would admit a unit Killing eigenfield $X$ corresponding to $\lambda_{i}$, i.e. such that $X \in l_{i}(G)$. Thus we would have $\lambda_{i}=\|\nabla X\|^{2}$ $\geq 0$, a contradiction. Analogously if $l_{i}(G) \neq\{0\}$ then $\lambda_{i} \geq 0$, a contradiction. Note that $\mathfrak{z}(\mathfrak{i s o}(M))=l_{1}(G) \oplus \ldots \oplus l_{k}(G)$ where $G=\operatorname{Iso}(M)$. Thus $\mathfrak{z}(\mathfrak{i s o}(M))=0$ and the result follows from Corollary 2 .

Note that a Lie group $G$ with a bi-invariant metric admits a $\operatorname{dim} G$ dimensional Lie algebra of Killing vector fields (right-invariant vector fields on $G$ ) with constant length. On the other hand we have:

Proposition 4. Assume that a simply connected homogeneous space $(M, g)$ admits a transitive group $G$ of isometries whose fundamental vector fields have constant length. Then $l_{G}=n=\operatorname{dim} M$ and $(M, g)$ is isometric to a Lie group $(G, m)$ with a bi-invariant metric. The space $(M, g)$ is a direct product $(M, g)=\left(G_{1}, m_{1}\right) \times \ldots \times\left(G_{k}, m_{k}\right)$ of simply connected Lie groups $G_{i}$ with bi-invariant Einstein metrics $m_{i}$. In particular $\nabla \varrho=0$.

Proof. It is easy to see that $\operatorname{dim} G=n$ and there exists a global orthonormal frame $\left\{E_{1}, \ldots, E_{n}\right\}$ of Killing vector fields $E_{i} \in \mathfrak{g}^{*}$ where $\mathfrak{g}^{*}$ denotes the Lie algebra of fundamental vector fields of the action of the group $G$. In fact, since $g(X, X)$ is constant for every $X \in \mathfrak{g}^{*}$ it follows that $g(X, Y)$ is constant for every $X, Y \in \mathfrak{g}^{*}$. Thus the linear mapping $F: \mathfrak{g}^{*} \rightarrow T_{x_{0}} M$ given by $F X=X_{x_{0}}$ for $X \in \mathfrak{g}^{*}$ is an isometry of $\mathfrak{g}^{*}$ with the induced scalar product onto $\left(T_{x_{0}} M, g_{x_{0}}\right)$. Note that $(M, g)$ is a g.o. space since the orbits of a one-parameter subgroup generated by a Killing vector field of constant length are geodesics. Since $(M, g)$ is simply connected it follows that $(M, g)$ is isometric to a simply connected Lie group $\widehat{G}$ with a left-invariant metric whose Lie algebra of right-invariant vector fields coincides with $\mathfrak{g}^{*}$. Hence $l(G)=l(\widehat{G})=n$ and $l(G)$ consists of left-invariant vector fields on $M=\widehat{G}$. 
Since right-invariant vector fields on $M$ have constant length it follows that a left-invariant metric on $M$ is bi-invariant.

Note that $l(G) \subset \mathfrak{i s o}(M)$ and each $l_{i}(G)$ is an ideal in the Lie algebra $l(G)$. Since $(M, g)$ is an $\mathcal{A}$-manifold and $L_{i}=\operatorname{ker}\left(S-\lambda_{i}\right.$ Id) are integrable it follows that all $L_{i}$ are auto-parallel. In addition $\left[l_{i}(G), l_{j}(G)\right]=0$ for $i \neq j$. Thus the almost product structure $T M=L_{1} \oplus \ldots \oplus L_{k}$ is integrable. Since the almost product structure $\left(L_{1}, \ldots, L_{k}\right)$ is integrable, the $L_{i}$ are parallel (see $[\mathrm{J}])$. The de Rham decomposition theorem yields that $(M, g)=$ $\left(G_{1}, m_{1}\right) \times \ldots \times\left(G_{k}, m_{k}\right)$ where each $G_{i}$ is a simply connected Lie group with Einstein bi-invariant metric $m_{i}$.

\section{Unimodular 4-dimensional Lie groups with almost Kähler} structures. Recall that a Lie algebra $\mathfrak{g}$ is called unimodular if $\operatorname{tr} \operatorname{ad}_{X}=0$ for all $X \in \mathfrak{g}$. Let $\mathfrak{g}$ be the Lie algebra of a Lie group $G$ and let $h$ be a positive definite scalar product on $\mathfrak{g}$. By a left-invariant metric on $G$ given by the scalar product $h$ we mean a metric $g \in \Gamma\left(T G^{*} \otimes T G^{*}\right)$ such that for any two left-invariant vector fields $X, Y \in \mathfrak{X}(G)$ and $x \in G$ we have $g(X, Y)_{x}=$ $h\left(X_{e}, Y_{e}\right)$ where $e \in G$ is the identity element of $G$ and where we identify $\mathfrak{g}$ with $T_{e} G$. In what follows we consider only left-invariant vector fields and we identify the metric $g$ with $h$. In particular if $\Phi \in \Gamma\left(T G \otimes T G^{*} \otimes T G^{*}\right)$ is a left-invariant tensor of type $(1,2)$ then if we take a left-invariant orthonormal frame on $G$ we get $\operatorname{tr}_{g} \Phi=\sum_{i=1}^{n} \Phi\left(E_{i}, E_{i}\right)=\sum \Phi_{\mid e}\left(E_{i e}, E_{i e}\right)=\operatorname{tr}_{h} \Phi_{\mid e}$. Recall that if $(G, g)$ is a Lie group with a left-invariant metric $g$ then for any left-invariant fields on $G$ we have the following formula (see e.g. [C-E]):

$$
\nabla_{X} Y=\frac{1}{2}\left([X, Y]-\operatorname{ad}_{X}^{*} Y-\operatorname{ad}_{Y}^{*} X\right)
$$

where $\nabla$ is the Levi-Civita connection of $(G, g)$ and $\operatorname{ad}_{X}^{*}$ denotes the operator adjoint to $\operatorname{ad}_{X}$ with respect to $g$, i.e. if $X, Y, Z$ are three left-invariant vector fields on $G$ then $g\left(\operatorname{ad}_{X}^{*} Y, Z\right)=g\left(Y, \operatorname{ad}_{X} Z\right)$. For any left-invariant vector field $X$ on $(G, g)$ let $A_{X}: T G \rightarrow T G$ be defined by $A_{X} Y=\nabla_{Y} X$. Note that $A_{X}: T G \ni Y \mapsto \nabla_{Y} X \in T G$ is a left-invariant operator and we define tensors ad : $T G \times T G \ni(X, Y) \mapsto \operatorname{ad}_{X} Y \in T G, \operatorname{ad}^{*}: T G \times T G \ni(X, Y) \mapsto$ $\operatorname{ad}_{X}^{*} Y \in T G$ as left-invariant extensions to $G$ of appropriate tensors defined on $\mathfrak{g}$.

LEMMA 1. Let $h$ be a scalar product on a Lie algebra $\mathfrak{g}$. Denote by $\mathrm{ad}_{X}^{*}$ the adjoint of $\operatorname{ad}_{X}$ with respect to $h$. Then $\mathfrak{g}$ is unimodular if and only if $\operatorname{tr}_{h} \operatorname{ad}_{X}^{*} Y=0$. Assume that $(G, g)$ is a unimodular Lie group with a leftinvariant metric $g$. Then $\operatorname{div} X=0$ for any left-invariant vector field $X$ on $G$, and if $X, Y$ are left-invariant vector fields on $G$, then

$$
\varrho(X, Y)=-\operatorname{tr} A_{X} \circ A_{Y} .
$$


Proof. Let $\left\{E_{1}, \ldots, E_{n}\right\}$ be any orthonormal basis of $(\mathfrak{g}, h)$. Note that for any $X \in \mathfrak{g}$ we have

$$
\begin{aligned}
h\left(\operatorname{tr}_{h} \operatorname{ad}^{*}, X\right) & =h\left(\sum_{i=1}^{n} \operatorname{ad}_{E_{i}}^{*} E_{i}, X\right)=\sum_{i=1}^{n} h\left(\operatorname{ad}_{E_{i}} X, E_{i}\right) \\
& =-\sum_{i=1}^{n} h\left(\operatorname{ad}_{X} E_{i}, E_{i}\right)=-\operatorname{tr} \operatorname{ad}_{X},
\end{aligned}
$$

and the first statement follows.

From (3.1) we obtain (set $\left.h=g_{\mid e}\right)$

$$
\begin{aligned}
\operatorname{div} X & =\operatorname{tr} A_{X}=\frac{1}{2}\left(\sum_{i=1}^{n} g\left(\operatorname{ad}_{E_{i}} X, E_{i}\right)-\sum_{i=1}^{n} g\left(\operatorname{ad}_{X} E_{i}, E_{i}\right)\right) \\
& =-\sum_{i=1}^{n} g\left(\operatorname{ad}_{X} E_{i}, E_{i}\right)=-\sum_{i=1}^{n} h\left(\operatorname{ad}_{X} E_{i}, E_{i}\right)=-\operatorname{trad} \operatorname{tr}_{X}
\end{aligned}
$$

and $\operatorname{div} X=-\operatorname{tr} \operatorname{ad}_{X}=0$. On the other hand for any vector field $X \in \mathfrak{X}(G)$ we have (see $[\mathrm{K}]$ )

$$
\varrho(X, X)=-\operatorname{tr} A_{X} \circ A_{X}+(\operatorname{div} X)^{2}-\operatorname{div}\left(A_{X} X\right)-\operatorname{div}((\operatorname{div} X) X) .
$$

Thus $\varrho(X, X)=-\operatorname{tr} A_{X} \circ A_{X}$ if $X$ is a left-invariant vector field, which proves $(3.2)$.

Proposition 5. Assume that a Lie group $(G, g)$ is an $\mathcal{A}$-manifold which has a generic Ricci tensor. Then $G$ is unimodular.

Proof. Since the result is local we can assume that $G$ is simply connected. Let $\left\{E_{1}, \ldots, E_{n}\right\}$ be an orthonormal basis of eigenfields of the Ricci tensor $S$ of $(G, g)$. Then the fields $E_{i}$ are left-invariant. Since $(G, g)$ is an $\mathcal{A}$-manifold we have $\nabla S\left(E_{i}, E_{i}\right)=0$ and $\nabla_{E_{i}} E_{i}=0$ for $i \in\{1, \ldots, n\}$. From (3.1) we obtain $\operatorname{ad}_{E_{i}}^{*} E_{i}=0$. Consequently, $\operatorname{tr}_{h}$ ad $^{*}=0$. Thus the result is clear in view of Lemma 1.

Now we consider the problem of existence of left-invariant almost Kähler structures on 4-dimensional Lie groups $(G, g)$. W. Thurston has given an example of an almost Kähler structure on a compact quotient of the (unique up to isomorphism) 4-dimensional simply connected non-commutative nilpotent Lie group $G$ with a left-invariant metric. This example also admits an opposite left-invariant almost Kähler structure. Every nilpotent group is unimodular with non-zero center. We generalize the above examples and give examples of 4-dimensional unimodular Lie groups with non-zero center which admit two mutually opposite left-invariant almost Kähler structures.

Proposition 6. Let $\mathfrak{g}$ be a 4-dimensional unimodular Lie algebra. Then a simply connected group with Lie algebra $\mathfrak{g}$ admits two mutually opposite 
left-invariant almost Kähler structures if and only if there exists a basis $\left\{E_{1}, E_{2}, E_{3}, E_{4}\right\}$ of $\mathfrak{g}$ such that $\left[E_{1}, E_{2}\right]=\left[E_{3}, E_{4}\right]=0$; and it admits a left-invariant almost Kähler structure if and only if there exists a basis $\left\{E_{1}, E_{2}, E_{3}, E_{4}\right\}$ of $\mathfrak{g}$ such that $\left[E_{1}, E_{2}\right]+\left[E_{3}, E_{4}\right]=0$.

Proof. Let $J$ be a left-invariant almost Hermitian structure on the Lie group $(G, g)$. Let $\left\{E_{1}, E_{2}, E_{3}, E_{4}\right\}$ be an orthonormal basis of $\mathfrak{g}$ such that

$$
J E_{1}=E_{2}, \quad J E_{3}=E_{4} .
$$

Note that $\delta J=-\operatorname{tr}_{g} \nabla J=-\sum \nabla J\left(E_{i}, E_{i}\right)$ and from (3.1) it follows that for any left-invariant vector field $X$ we have $\nabla_{X} X=-\operatorname{ad}_{X}^{*} X$. Thus we obtain

$$
\begin{aligned}
& \nabla J\left(E_{1}, E_{1}\right)+J\left(\nabla_{E_{1}} E_{1}\right)=\nabla_{E_{1}} E_{2}, \\
& \nabla J\left(E_{2}, E_{2}\right)+J\left(\nabla_{E_{2}} E_{2}\right)=-\nabla_{E_{2}} E_{1}, \\
& \nabla J\left(E_{3}, E_{3}\right)+J\left(\nabla_{E_{3}} E_{3}\right)=\nabla_{E_{3}} E_{4}, \\
& \nabla J\left(E_{4}, E_{4}\right)+J\left(\nabla_{E_{4}} E_{4}\right)=-\nabla_{E_{4}} E_{3},
\end{aligned}
$$

and

(3.3a) $\nabla J\left(E_{1}, E_{1}\right)+\nabla J\left(E_{2}, E_{2}\right)=J\left(\operatorname{ad}_{E_{1}}^{*} E_{1}\right)+J\left(\operatorname{ad}_{E_{2}}^{*} E_{2}\right)+\left[E_{1}, E_{2}\right]$,

(3.3b) $\nabla J\left(E_{3}, E_{3}\right)+\nabla J\left(E_{4}, E_{4}\right)=J\left(\operatorname{ad}_{E_{3}}^{*} E_{3}\right)+J\left(\operatorname{ad}_{E_{4}}^{*} E_{4}\right)+\left[E_{3}, E_{4}\right]$.

Consequently, $\delta J=-\operatorname{tr}_{g} \nabla J=-J\left(\operatorname{tr}_{g} \mathrm{ad}^{*}\right)-\left[E_{1}, E_{2}\right]-\left[E_{3}, E_{4}\right]=$ $-\left[E_{1}, E_{2}\right]-\left[E_{3}, E_{4}\right]$. It follows (see (1.2)) that $J$ is almost Kähler if and only if $\left[E_{1}, E_{2}\right]+\left[E_{3}, E_{4}\right]=0$.

Now assume that $(G, g)$ admits two mutually opposite left-invariant almost Kähler structures $J, \bar{J}$. Then there exists an orthonormal basis $\left\{E_{1}, E_{2}\right.$, $\left.E_{3}, E_{4}\right\}$ of left-invariant vector fields of $\mathfrak{g}$ such that

$$
J E_{1}=E_{2}, \quad J E_{3}=E_{4}, \quad \bar{J} E_{1}=E_{2}, \quad \bar{J} E_{3}=-E_{4} .
$$

Consequently, $\left[E_{1}, E_{2}\right]+\left[E_{3}, E_{4}\right]=-\delta J=0,\left[E_{1}, E_{2}\right]-\left[E_{3}, E_{4}\right]=-\delta \bar{J}=0$ and $\left[E_{1}, E_{2}\right]=0,\left[E_{3}, E_{4}\right]=0$. On the other hand, if there exists a basis $\left\{E_{1}, E_{2}, E_{3}, E_{4}\right\}$ of a unimodular Lie algebra $\mathfrak{g}$ satisfying one of the above two conditions then it is easy to construct almost Kähler structures on $G$ by the above formulas. In fact, take a left-invariant metric $g$ on $G$ such that $\left\{E_{1}, E_{2}, E_{3}, E_{4}\right\}$ is an orthonormal frame with respect to $g$. Define an almost Hermitian structure $J$ on $G$ by (*) or two mutually opposite almost Hermitian structures by $(* *)$ respectively. Since equations (3.3) are satisfied and $\operatorname{tr}_{h} \mathrm{ad}^{*}=0$ it follows that $\delta J=-\left[E_{1}, E_{2}\right]-\left[E_{3}, E_{4}\right]=0$ in the first case and analogously $\delta J=\delta \bar{J}=0$ in the second case, which means that the almost Hermitian structures considered are almost Kähler.

Now we prove 
Proposition 7. Let $(\mathfrak{g},[]$,$) be a unimodular Lie algebra and \operatorname{dim} \mathfrak{g}=4$. Assume that $\operatorname{dim}[\mathfrak{g}, \mathfrak{g}] \leq 2$ and $G$ is a simply connected Lie group with Lie algebra $\mathfrak{g}$. Then $G$ is solvable and every left-invariant metric on $G$ admits two mutually opposite invariant compatible almost Kähler structures.

Proof. Since $[\mathfrak{g}, \mathfrak{g}]$ is at most two-dimensional it is clear that $G$ is solvable. Let $g$ be a left-invariant metric on $G$ and let $h$ be the corresponding scalar product on $\mathfrak{g}$. We denote by $\Lambda^{+} \mathfrak{g}$ (resp. $\Lambda^{-} \mathfrak{g}$ ) the space of self-dual (resp. anti-self-dual) 2-forms on $\mathfrak{g}$ (with respect to $h$ ). Define an endomorphism $S: \bigwedge^{2} \mathfrak{g} \rightarrow[\mathfrak{g}, \mathfrak{g}]$ as follows: if $\omega=X \wedge Y$ is a simple bivector then $S(\omega)=$ $[X, Y]$. Then $S$ is well defined on the whole of $\bigwedge^{2} \mathfrak{g}$ and $\operatorname{im} S=[\mathfrak{g}, \mathfrak{g}]$. Now set $S_{+}=\left.S\right|_{\Lambda^{+} \mathfrak{g}}$ and $S_{-}=\left.S\right|_{\Lambda^{-} \mathfrak{g}}$. Note that $\operatorname{im} S_{ \pm} \subset[\mathfrak{g}, \mathfrak{g}]$. Since $\operatorname{dim} \Lambda^{ \pm} \mathfrak{g}=3$ it follows that there exist forms $\Phi \in \Lambda^{+} \mathfrak{g}$ and $\Psi \in \Lambda^{-} \mathfrak{g}$ such that $S_{+} \Phi=0$, $S_{-} \Psi=0$ and $\|\Phi\|=\|\Psi\|=\sqrt{2}$. It is well known that if $\Phi \in \bigwedge^{2} \mathfrak{g}$ is a non-zero self-dual form then there exists an oriented orthonormal basis $\left\{E_{1}, E_{2}, E_{3}, E_{4}\right\}$ of $\mathfrak{g}$ and a real number $\alpha>0$ such that $\Phi=\alpha\left(E_{1} \wedge E_{2}+\right.$ $\left.E_{3} \wedge E_{4}\right)$ and $\|\Phi\|=\sqrt{2} \alpha$. Thus, since $\|\Phi\|=\sqrt{2}$ we have $\Phi=E_{1} \wedge E_{2}+$ $E_{3} \wedge E_{4}$ and

$$
S_{+}(\Phi)=S\left(E_{1} \wedge E_{2}+E_{3} \wedge E_{4}\right)=\left[E_{1}, E_{2}\right]+\left[E_{3}, E_{4}\right]=0 .
$$

Consequently, the almost Hermitian structure $J$ on $G$ given by $(*)$ is an almost Kähler structure in view of Proposition 6. Analogously $\Psi$ gives an almost Kähler structure $\bar{J}$ which is opposite to $J$.

Let us define a Lie algebra $\mathfrak{g}=\mathfrak{g}_{\{a, b, c, d, f\}}$ as follows: $\mathfrak{g}=\operatorname{span}\left\{E_{1}, E_{2}\right.$, $\left.E_{3}, E_{4}\right\}$ with the Lie bracket

$$
\begin{aligned}
& {\left[E_{1}, E_{2}\right]=0, \quad\left[E_{1}, E_{3}\right]=0, \quad\left[E_{1}, E_{4}\right]=0,} \\
& {\left[E_{2}, E_{3}\right]=a E_{1}+b E_{4}+f E_{3}, \quad\left[E_{2}, E_{4}\right]=c E_{1}+d E_{3}-f E_{4},} \\
& {\left[E_{3}, E_{4}\right]=0 .}
\end{aligned}
$$

Note that some of the algebras $\mathfrak{g}=\mathfrak{g}_{\{a, b, c, d, f\}}$ are isomorphic to others of this form. It is easy to check that $\mathfrak{g}$ is a unimodular solvable Lie algebra $([\mathfrak{g}, \mathfrak{g}]$ is an at most two-dimensional commutative algebra). Take a simply connected Lie group $G_{\{a, b, c, d, f\}}$ with Lie algebra $\mathfrak{g}_{\{a, b, c, d, f\}}$. Note that every group $\left(G_{\{a, b, c, d, f\}}, g\right)$ admits two mutually opposite left-invariant almost Kähler structures $J, \bar{J}$ such that $J E_{1}=E_{2}, J E_{3}=E_{4}, \bar{J} E_{1}=E_{2}, \bar{J} E_{3}=-E_{4}$. The basis $\left\{E_{1}, E_{2}, E_{3}, E_{4}\right\}$ defines a left-invariant metric $g$ on $G=G_{\{a, b, c, d, f\}}$ such that $\left\{E_{1}, E_{2}, E_{3}, E_{4}\right\}$ is an orthonormal frame on $(G, g)$. Then in the basis $\left\{E_{1}, E_{2}, E_{3}, E_{4}\right\}$ we have

$$
\operatorname{ad}_{E_{1}}^{*}=\left(\begin{array}{cccc}
0 & 0 & 0 & 0 \\
0 & 0 & 0 & 0 \\
0 & 0 & 0 & 0 \\
0 & 0 & 0 & 0
\end{array}\right), \quad \operatorname{ad}_{E_{2}}^{*}=\left(\begin{array}{cccc}
0 & 0 & 0 & 0 \\
0 & 0 & 0 & 0 \\
a & 0 & f & b \\
c & 0 & d & -f
\end{array}\right),
$$




$$
\operatorname{ad}_{E_{3}}^{*}=\left(\begin{array}{cccc}
0 & 0 & 0 & 0 \\
-a & 0 & -f & -b \\
0 & 0 & 0 & 0 \\
0 & 0 & 0 & 0
\end{array}\right), \quad \operatorname{ad}_{E_{4}}^{*}=\left(\begin{array}{cccc}
0 & 0 & 0 & 0 \\
-c & 0 & -d & f \\
0 & 0 & 0 & 0 \\
0 & 0 & 0 & 0
\end{array}\right) .
$$

Consequently, we obtain (setting $A_{E_{i}}=\nabla E_{i}$ )

$$
\begin{array}{rlrl}
A_{E_{1}} & =\left(\begin{array}{cccc}
0 & 0 & 0 & 0 \\
0 & 0 & \frac{a}{2} & \frac{c}{2} \\
0 & -\frac{a}{2} & 0 & 0 \\
0 & -\frac{c}{2} & 0 & 0
\end{array}\right), & A_{E_{2}}=\left(\begin{array}{cccc}
0 & 0 & -\frac{a}{2} & -\frac{c}{2} \\
0 & 0 & 0 & 0 \\
-\frac{a}{2} & 0 & -f & -\frac{b+d}{2} \\
-\frac{c}{2} & 0 & -\frac{b+d}{2} & f
\end{array}\right), \\
A_{E_{3}}=\left(\begin{array}{cccc}
0 & \frac{a}{2} & 0 & 0 \\
\frac{a}{2} & 0 & f & \frac{d+b}{2} \\
0 & 0 & 0 & 0 \\
0 & \frac{b-d}{2} & 0 & 0
\end{array}\right), & A_{E_{4}}=\left(\begin{array}{cccc}
0 & \frac{c}{2} & 0 & 0 \\
\frac{c}{2} & 0 & \frac{b+d}{2} & -f \\
0 & \frac{d-b}{2} & 0 & 0 \\
0 & 0 & 0 & 0
\end{array}\right) .
\end{array}
$$

For the Ricci tensor $\varrho$ of $(G, g)$ we have the formula $\varrho\left(E_{i}, E_{j}\right)=-\operatorname{tr} A_{E_{i}} \circ$ $A_{E_{j}}$. Thus we get

$$
\begin{gathered}
\varrho\left(E_{1}, E_{2}\right)=0, \quad \varrho\left(E_{1}, E_{3}\right)=\frac{c d}{2}+\frac{a f}{2}, \quad \varrho\left(E_{1}, E_{4}\right)=\frac{a b}{2}-\frac{c f}{2}, \\
\varrho\left(E_{2}, E_{3}\right)=0, \quad \varrho\left(E_{2}, E_{4}\right)=0, \quad \varrho\left(E_{3}, E_{4}\right)=-\frac{a c}{2}+f(b-d), \\
\varrho\left(E_{1}, E_{1}\right)=\frac{1}{2}\left(a^{2}+c^{2}\right), \quad \varrho\left(E_{2}, E_{2}\right)=-\frac{1}{2}\left(a^{2}+c^{2}+2 f^{2}+(d+b)^{2}\right), \\
\varrho\left(E_{3}, E_{3}\right)=-\frac{1}{2}\left(a^{2}+b^{2}-d^{2}\right), \quad \varrho\left(E_{4}, E_{4}\right)=-\frac{1}{2}\left(c^{2}+d^{2}-b^{2}\right) .
\end{gathered}
$$

Now we give several interesting special cases of manifolds $\left(G_{\{a, b, c, d, f\}}, g\right)$. Note that the fields $\left\{E_{1}, E_{2}, E_{3}, E_{4}\right\}$ are eigenfields of $\varrho$ if one of the following three cases holds:
(a) $a=d=f=0$,
(a1) $c=b=f=0$,
(b) $a=c=f=0$.

Note that in the first case we get an example of a Lie group with generic Ricci tensor which admits two mutually opposite almost Kähler non-Kähler structures; in this case $\lambda_{1}=c^{2} / 2, \lambda_{2}=-c^{2} / 2-b^{2} / 2, \lambda_{3}=$ $-b^{2} / 2, \lambda_{4}=-c^{2} / 2+b^{2} / 2$ and thus if $c \neq 0, b \neq 0, b^{2} \neq 2 c^{2}, 2 b^{2} \neq c^{2}$ then the eigenvalues $\lambda_{i}$ of $\varrho$ are all distinct. Similarly in case (b) we get $\lambda_{1}=0, \lambda_{2}=-(d+b)^{2} / 2, \lambda_{3}=-\left(b^{2}-d^{2}\right) / 2, \lambda_{4}=-\left(d^{2}-b^{2}\right) / 2$. Thus if $|d| \neq|b|, b d \neq 0$ then $\left(G_{\{0, b, 0, d, 0\}}, g\right)$ has a generic Ricci tensor and admits two mutually opposite left-invariant almost Kähler structures. In case (a), if $c=0$ then we get Thurston's example of an almost Kähler structure on a nilpotent 4 -dimensional Lie group. In this case the Lie algebra $\mathfrak{g}$ is nilpotent with two-dimensional center $\mathfrak{z}$, hence in fact it admits a circle 
of almost Kähler structures in both orientations. If $\mathfrak{z}=\operatorname{span}\left\{F_{1}, F_{2}\right\}$ and $\mathfrak{m}=\mathfrak{z}^{\perp}=\operatorname{span}\left\{F_{3}, F_{4}\right\}$ where $F_{1}, F_{2}$ (resp. $F_{3}, F_{4}$ ) is any orthonormal basis of $\mathfrak{z}$ (resp. of $\mathfrak{m}$ ) then the almost Hermitian structures $J_{1}, J_{2}$ defined by $J_{1} F_{1}=F_{2}, J_{1} F_{3}=F_{4}, J_{2} F_{1}=F_{3}, J_{2} F_{2}=-F_{4}$ are almost Kähler. It is easy to see that $J_{\phi}=\cos \phi J_{1}+\sin \phi J_{2}$ is a strictly almost Kähler structure for $\phi \in \mathbb{R}$. Thurston's example also admits two mutually opposite Hermitian structures (see $[\mathrm{S}]$ ). Note that any left-invariant metric on the Thurston example admits two circles of mutually opposite almost Kähler structures and two mutually opposite Hermitian structures.

Let us now return to case (b). If we take $b=-\varepsilon d=1$ where $\varepsilon \in\{-1,1\}$ then the Lie algebra $\mathfrak{g}=\mathfrak{g}_{\{0,1,0,-\varepsilon, 0\}}$ is

$$
\begin{array}{lll}
{\left[E_{1}, E_{2}\right]=0,} & {\left[E_{1}, E_{3}\right]=0,} & {\left[E_{1}, E_{4}\right]=0,} \\
{\left[E_{2}, E_{3}\right]=E_{4},} & {\left[E_{2}, E_{4}\right]=-\varepsilon E_{3},} & {\left[E_{3}, E_{4}\right]=0 .}
\end{array}
$$

The simply connected solvable Lie groups $G_{\varepsilon}$ corresponding to $\mathfrak{g}=$ $\mathfrak{g}_{\{0,1,0,-\varepsilon, 0\}}$ are $G_{1}=\mathbb{R} \times \widetilde{E(2)}$ and $G_{-1}=\mathbb{R} \times E(1,1)$ where $\widetilde{E(2)}$ is a universal covering of the Lie group of oriented isometries of the Euclidean space $E^{2}, E(2)=\mathrm{SO}(2) \bowtie \mathbb{R}^{2}$ and the group $E(1,1)=\mathrm{SO}(1,1) \bowtie \mathbb{R}^{2}$ is the group of oriented isometries of the Lorentz space $E^{(1,1)}$ with scalar product of signature $(+,-)$. The Lie groups $E(2)$ and $E(1,1)$ can be represented by the following subgroups of $\mathrm{GL}(3, \mathbb{R})$ :

$$
E(2)=\left\{\left(\begin{array}{ccc}
\cos \phi & \sin \phi & a \\
-\sin \phi & \cos \phi & b \\
0 & 0 & 1
\end{array}\right)\right\}, \quad E(1,1)=\left\{\left(\begin{array}{ccc}
\cosh \phi & \sinh \phi & a \\
-\sinh \phi & \cosh \phi & b \\
0 & 0 & 1
\end{array}\right)\right\}
$$

where $\phi, a, b \in \mathbb{R}$. The Lie algebra $\mathfrak{g}=\mathfrak{g}_{\{0,1,0,-1,0\}}$ can be realized as a Lie subalgebra of the Lie algebra of smooth vector fields on the torus $T^{4}$. In fact we have $T^{4}=\mathbb{R}^{4} / \Gamma_{0}$ where $\Gamma_{0}=2 \pi \mathbb{Z} \oplus 2 \pi \mathbb{Z} \oplus 2 \pi \mathbb{Z} \oplus 2 \pi \mathbb{Z} \subset \mathbb{R}^{4}$. Define vector fields $E_{i}$ by

$$
\begin{array}{ll}
E_{1}=\frac{\partial}{\partial x_{1}}, & E_{3}=\cos x_{2} \frac{\partial}{\partial x_{3}}+\sin x_{2} \frac{\partial}{\partial x_{4}}, \\
E_{2}=\frac{\partial}{\partial x_{2}}, & E_{4}=-\sin x_{2} \frac{\partial}{\partial x_{3}}+\cos x_{2} \frac{\partial}{\partial x_{4}}
\end{array}
$$

where $\left\{\partial / \partial x_{1}, \partial / \partial x_{2}, \partial / \partial x_{3}, \partial / \partial x_{4}\right\}$ is the standard parallel frame on $T^{4}$ with standard flat metric. Then we have relations (3.4) with $\varepsilon=1$. Note that then an orthonormal coframe $\left\{\theta_{1}, \theta_{2}, \theta_{3}, \theta_{4}\right\}$ corresponding to the (flat) leftinvariant metric given by the fields $\left\{E_{1}, E_{2}, E_{3}, E_{4}\right\}$ is $\theta_{1}=d x_{1}, \theta_{2}=d x_{2}$, $\theta_{3}=\cos x_{2} d x_{3}+\sin x_{2} d x_{4}, \theta_{4}=-\sin x_{2} d x_{3}+\cos x_{2} d x_{4}$. It follows that on the universal covering space $\mathbb{R}^{4}$ of $T^{4}$ there exists a structure of a solvable 
Lie group $G$ with Lie algebra generated by the lifts $E_{i}^{*}$ of the fields $E_{i}$. $G$ is in fact a universal covering group of the Lie group $S^{1} \times E(2)$. Define the fields $F_{i}$ as follows: $F_{1}=E_{1}, F_{2}=E_{2}, F_{3}=b E_{3}, F_{4}=E_{4}$ where the $E_{i}$ satisfy (3.5). Then

$$
\begin{aligned}
& {\left[F_{1}, F_{2}\right]=0, \quad\left[F_{1}, F_{3}\right]=0, \quad\left[F_{1}, F_{4}\right]=0,} \\
& {\left[F_{2}, F_{3}\right]=b F_{4}, \quad\left[F_{2}, F_{4}\right]=-\varepsilon \frac{1}{b} F_{3}, \quad\left[F_{3}, F_{4}\right]=0 .}
\end{aligned}
$$

It follows that $T^{4}$ admits a family $\left\{g_{b}\right\}, b \in \mathbb{R}_{+}-\{1\}$, of locally homogeneous metrics with generic Ricci tensor with two compatible (locally) invariant strictly almost Kähler structures. An orthonormal coframe $\left\{\bar{\theta}_{1}, \bar{\theta}_{2}, \bar{\theta}_{3}, \bar{\theta}_{4}\right\}$ corresponding to the left-invariant metric given by the fields $\left\{F_{1}, F_{2}, F_{3}, F_{4}\right\}$ is $\bar{\theta}_{1}=\theta_{1}, \bar{\theta}_{2}=\theta_{2}, \bar{\theta}_{3}=\frac{1}{b} \theta_{3}, \bar{\theta}_{4}=\theta_{4}$. The eigenvalues of the Ricci tensor are

$$
\lambda_{1}=0, \quad \lambda_{2}=-\frac{1}{2}\left(b-\frac{1}{b}\right)^{2}, \quad \lambda_{3}=-\frac{1}{2}\left(b^{2}-\frac{1}{b^{2}}\right), \quad \lambda_{4}=-\frac{1}{2}\left(\frac{1}{b^{2}}-b^{2}\right) .
$$

The Kähler form of $\left(T^{4}, g_{b}, J_{ \pm}\right)$is

$$
\Omega_{b}=d x_{1} \wedge d x_{2} \pm \frac{1}{b} d x_{3} \wedge d x_{4}
$$

and the metric is

$$
\begin{aligned}
g_{b}= & \left(d x_{1}\right)^{2}+\left(d x_{2}\right)^{2}+\left(\frac{1}{b^{2}}\left(\cos x_{2}\right)^{2}+\left(\sin x_{2}\right)^{2}\right)\left(d x_{3}\right)^{2} \\
& +\left(1-\frac{1}{b^{2}}\right) \sin 2 x_{2} d x_{3} \odot d x_{4} \\
& +\left(\frac{1}{b^{2}}\left(\sin x_{2}\right)^{2}+\left(\cos x_{2}\right)^{2}\right)\left(d x_{4}\right)^{2} .
\end{aligned}
$$

It is known that both groups $E(2), E(1,1)$ admit discrete uniform subgroups $\Gamma$, i.e. such that $N_{\Gamma}=\Gamma \backslash G$ are compact spaces and $G=E(2)$ or $G=$ $E(2,2)$ (for an interesting exposition of the geometry of 3-manifolds see [Sc], [O-T]). It follows that the compact spaces $M_{\Gamma}=S^{1} \times N_{\Gamma}$ admit a family of homogeneous metrics $\left\{g_{b}: b \in \mathbb{R}_{+}-\{1\}\right\}$ such that $\left(M_{\Gamma}, g_{b}\right)$ is a compact homogeneous space with two mutually opposite invariant almost Kähler structures and generic Ricci tensor with eigenvalues

$$
\lambda_{1}=0, \quad \lambda_{2}=-\frac{1}{2}\left(b-\varepsilon \frac{1}{b}\right)^{2}, \quad \lambda_{3}=-\frac{1}{2}\left(b^{2}-\frac{1}{b^{2}}\right), \quad \lambda_{4}=-\frac{1}{2}\left(\frac{1}{b^{2}}-b^{2}\right) .
$$

REMARK. The symplectic structure of the solvmanifolds $M_{\Gamma}=S^{1} \times N_{\Gamma}$ from the viewpoint of symplectic geometry has already been investigated (see for example [O-T]). However, as far as the author knows, almost Kähler metrics on these manifolds have not been studied. 
Acknowledgements. The author is grateful to the referee for his useful remarks which made the paper more readable. The work was supported by KBN grant 2 P0 3A 01615.

\section{References}

[B] A. L. Besse, Einstein Manifolds, Springer, Berlin, 1987.

[B-V] P. Bueken and L. Vanhecke, Three and four-dimensional Einstein-like manifolds and homogeneity, Geom. Dedicata 75 (1999), 123-136.

[C-E] J. Cheeger and D. G. Ebin, Comparison Theorems in Riemannian Geometry, North-Holland, Amsterdam, 1975.

[C-S-V] J. T. Cho, K. Sekigawa and L. Vanhecke, Volume-preserving geodesic symmetries on four-dimensional Hermitian Einstein spaces, Nagoya Math. J. 146 (1997), 13-29.

[C-F-L] L. Cordero, M. Fernández and M. de León, Examples of compact non-Kähler almost Kähler manifolds, Proc. Amer. Math. Soc. 95 (1985), 280-286.

[De] A. Derdziński, Self-dual Kähler manifolds and Einstein manifolds of dimension four, Compositio Math. 49 (1983), 405-433.

[D] T. Drăghici, The Kähler cone versus the symplectic cone, Bull. Math. Soc. Sci. Math. Roumanie 42 (90) (1999), 41-49.

[F-G] M. Fernández, M. J. Gotay and A. Gray, Compact parallelizable four-dimensional symplectic and complex manifolds, Proc. Amer. Math. Soc. 103 (1988), 1209-1212.

[G] A. Gray, Einstein-like manifolds which are not Einstein, Geom. Dedicata 7 (1978), 259-280.

[J] W. Jelonek, Killing tensors and warped products, Ann. Polon. Math. 75 (2000), $15-33$.

[Ka] A. Kaplan, On the geometry of groups of Heisenberg type, Bull. London Math. Soc. 15 (1983), 35-42.

[K] S. Kobayashi, Transformations Groups in Differential Geometry, Springer, Berlin, 1972.

[K-N] S. Kobayashi and K. Nomizu, Foundations of Differential Geometry, Interscience Publ., New York, 1963.

[Kod] K. Kodaira, On the structure of compact complex analytic surfaces I, Amer. J. Math. 86 (1964), 751-798.

[K-V] O. Kowalski and L. Vanhecke, Riemannian manifolds with homogeneous geodesics, Boll. Un. Mat. Ital. B (7) 5 (1991), 189-246.

[O-S] T. Oguro and K. Sekigawa, Almost Kähler structures on the Riemannian product of a 3-dimensional hyperbolic space and a real line, Tsukuba J. Math. 20 (1996), $151-161$.

[O-T] J. Oprea and A. Tralle, Symplectic Manifolds with no Kähler Structure, Springer, 1997.

[S] S. Salamon, Special structures on four manifolds, Riv. Mat. Univ. Parma (4) 17 (1991), 109-123.

[Sc] P. Scott, The geometries of 3-manifolds, Bull. London Math. Soc. (5) 15 (1983), 401-487.

[Se-1] K. Sekigawa, On some 4-dimensional compact Einstein almost Kähler manifolds, Math. Ann. 271 (1985), 333-337. 
[Se-2] K. Sekigawa, On some compact Einstein almost Kähler manifolds, J. Math. Soc. Japan 39 (1987), 677-684.

[T] W. P. Thurston, Some simple examples of symplectic manifolds, Proc. Amer. Math. Soc. 55 (1976), 467-468.

[T-V] F. Tricerri and L. Vanhecke, Homogeneous Structures on Riemannian Manifolds, Cambridge Univ. Press, Cambridge, 1983.

Institute of Mathematics

Technical University of Cracow

Warszawska 24

31-155 Kraków, Poland

E-mail: wjelon@usk.pk.edu.pl
Institute of Mathematics Polish Academy of Sciences

Cracow Branch

Św. Tomasza 30

31-027 Kraków, Poland

Reçu par la Rédaction le 20.12.2000

Révisé le 14.5.2001 